\title{
Human fronto-tectal and fronto-striatal-tectal pathways activate differently during anti-saccades
}

\author{
Antoin D. de Weijer, Rene C. W. Mandl, Iris E. C. Sommer, Matthijs Vink, Rene S. Kahn \\ and Sebastiaan F.W. Neggers*
}

Department of Psychiatry, Rudolf Magnus Institute of Neuroscience, University Medical Center Utrecht, Utrecht, Netherlands

\section{Edited by:}

Chris Rorden, University of South

Carolina, USA

\section{Reviewed by:}

Tony Ro, City College of New York, USA

Paul Corballis, Georgia Institute of

Technology, USA

*Correspondence:

Sebastiaan F.W. Neggers, Department of Psychiatry, Rudolf Magnus Institute for Neuroscience, University Medical Center Utrecht, Heidelberglaan 100, Room B. 01.103, 3584 CX Utrecht, Netherlands.

e-mail:b.neggers@umcutrecht.nl
Almost all cortical areas in the vertebrate brain take part in recurrent connections through the subcortical basal ganglia (BG) nuclei, through parallel inhibitory and excitatory loops. It has been suggested that these circuits can modulate our reactions to external events such that appropriate reactions are chosen from many available options, thereby imposing volitional control over behavior. The saccade system is an excellent model system to study cortico-BG interactions. In this study two possible pathways were investigated that might regulate automaticity of eye movements in the human brain; the cortico-tectal pathway, running directly between the frontal eye fields (FEF) and superior colliculus (SC) and the cortico-striatal pathway from the FEF to the SC involving the caudate nucleus (CN) in the BG. In an event-related functional magnetic resonance imaging (fMRI) paradigm participants made pro- and anti-saccades. A diffusion tensor imaging (DTI) scan was made for reconstruction of white matter tracts between the FEF, CN and SC. DTI fiber tracts were used to divide both the left and right FEF into two sub-areas, projecting to either ipsilateral SC or CN. For each of these FEF zones an event-related fMRI timecourse was extracted. In general activity in the FEF was larger for anti-saccades. This increase in activity was lateralized with respect to anti-saccade direction in FEF zones connected to the SC but not for zones only connected to the $\mathrm{CN}$. These findings suggest that activity along the contralateral FEF-SC projection is responsible for directly generating anti-saccades, whereas the pathway through the BG might merely have a gating function withholding or allowing a pro-saccade.

Keywords: frontal eye fields, superior colliculus, caudate nucleus, anti-saccades, DTI, fMRI

\section{INTRODUCTION}

When we react to events in our direct environment, the proper reaction often depends on the context of the situation. For example, when a cup falls from the table, we would normally grab it automatically. On the other hand, when the cup contains boiling hot coffee, we might choose to withdraw our hand to prevent a burn.

Single-neuron recordings on non-human primates suggest that higher cortical areas control purposeful behavior, whereas subcortical areas are responsible for automatic reactions. Multiple parallel excitatory and inhibitory loops, from a large number of cortical areas through the basal ganglia (BG), modulate cortical activity and therefore might subserve many functions such as eye and bodily movements and even higher cognitive processes (Alexander and Crutcher, 1990).

The BG neuroanatomy is thus well suited to select one reaction over others (Redgrave et al., 1999). The neurophysiology underlying the ability to select the proper reaction in a certain context has often been studied using eye movements as a model system. The pro- and anti-saccade paradigm requires participants to either look toward or away from an emerging target and has proven to be an ideal behavioral paradigm to study our control over response selection (Munoz and Everling, 2004).

From a large body of work on non-human primates a network dedicated to saccade control has been identified in which the frontal cortex exerts influence over eye movement generation through connections to the SC, some direct and some through the BG nuclei (Munoz and Everling, 2004). The cortical frontal and supplementary eye fields (FEF/SEF) and lateral-intra-parietal eye fields contribute to saccadic eye movement generation and project directly to the midbrain oculomotor structure superior colliculus (SC) through the cortico-tectal tract (Segraves et al., 1987). The SC is the main gateway to the oculomotor nuclei in the brainstem that directly control the extra-ocular eye muscles (Sparks, 2002).

Projections from the FEF/SEF to the SC are relayed through the BG in two parallel pathways using multiple inhibitory synapses (Hikosaka et al., 2000), see also Figure 1. The excitatory direct pathway starts with projections from the FEF to the caudate nucleus (CN) that inhibit the substantia nigra pars reticulata (SNpr) that itself normally tonically inhibits the SC. Therefore the direct pathway provides a net excitatory influence of the FEF over the $\mathrm{SC}$ as tonic inhibition of the $\mathrm{SC}$ is released when the pathway is activated. The inhibitory indirect pathway between FEF and SC through the $\mathrm{BG}$ runs from the FEF/SEF to the $\mathrm{CN}$, the external segment of the globus pallidus (GPe), the sub-thalamic nucleus (STN), the SNpr, and finally the SC (Munoz and Everling, 2004). Although it was suggested that this pathway might prevent inappropriate automatic eye movements evoked by the SC (Munoz and Everling, 2004), many uncertainties remain how this complex system controls purposeful eye movements. 


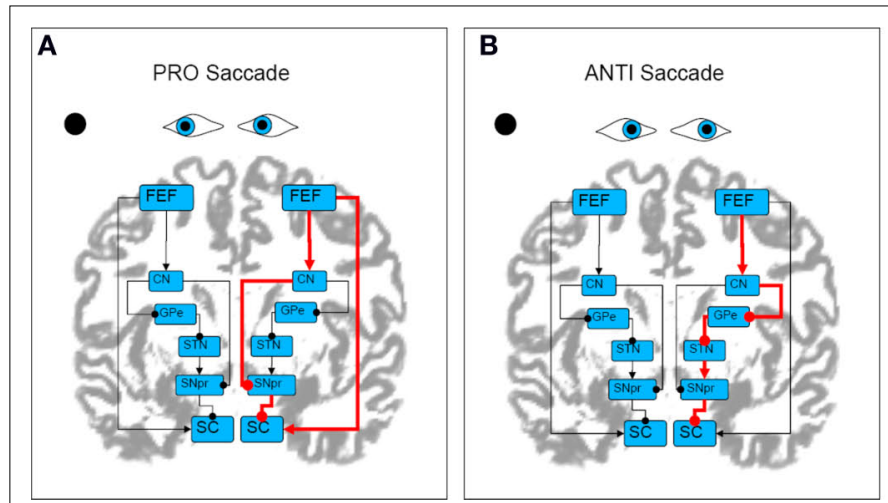

FIGURE 1 | Possible mechanisms for the FEF to regulate pro- and anti-saccade generation. The target is displayed as a black dot. For prosaccades, the FEF contralateral to saccade direction can send a motor command directly to the SC in the same hemisphere and/or through the CN > SNpr (the so called 'direct pathway') (A). For anti-saccades, more scenarios are possible: the FEF ipsilateral to saccade direction could
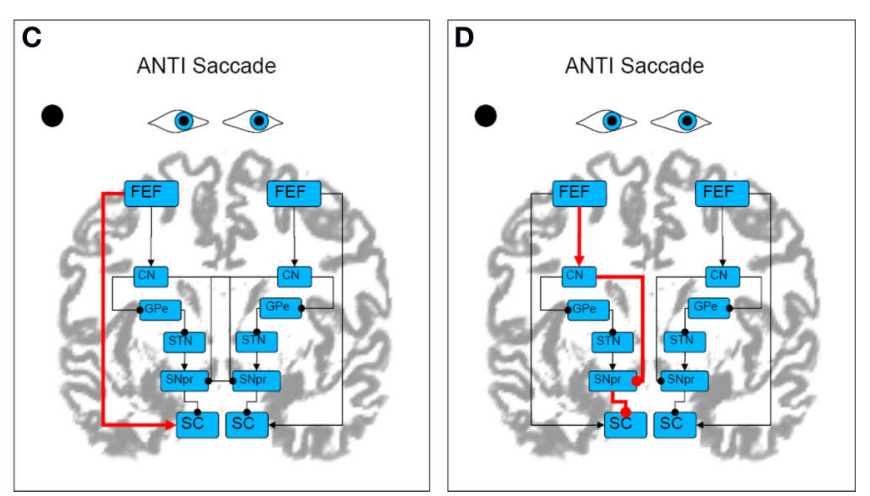

actively inhibition ipsilateral SC (through the 'indirect pathway') (B), thus inhibiting a pro-saccade. The FEF contralateral to saccade direction could excite the SC in the same hemisphere directly (C) or through the $\mathrm{CN}>\mathrm{GPe}>\mathrm{SNpr}$ ('direct') pathway (D), enforcing an anti-saccade over a pro-saccade. Also, a combination of scenario (B-D) could underly anti-saccade generation.
In the light of the above, there are three (not mutually exclusive) scenarios to correctlygenerate an anti-saccade, see also Figures 1B-D. First, the indirect pathway through the BG ipsilateral to saccade direction could be used to inhibit the ipsilateral SC and hence prevent it from executing an automatic pro-saccade to the target (Figure 1B). In that case, the signal from the FEF into the indirect pathway would be lateralized in an ipsilateral fashion with respect to saccade direction (higher FEF activation for ipsilateral saccades). Second, the direct pathway from the FEF through the BG contralateral to saccade direction could receive more input to disinhibit the SC contralateral to saccade direction (Figure 1D) and have it generate an anti-saccade. If so, the signal from the FEF into the direct pathway would be lateralized in a contralateral fashion with respect to saccade direction (higher activation for contralateral saccades in the part of the FEF connected to the direct pathway). Third, the projections from the FEF directly to the SC contralateral to saccade direction, bypassing the $\mathrm{BG}$, could be enhanced to overcome an automatic pro-saccade that would otherwise have been executed (Figure 1D). If the latter mechanism is driving anti-saccade production, the signal coming from FEF neurons connected directly to the SC should be lateralized in a contralateral fashion with respect to saccade direction. Also, a combination of the aforementioned pathways could be used to generate anti-saccades, i.e. they are not mutually exclusive.

Hardly any in vivo human connectivity or functional imaging reports exist that deal with how cortico-BG and cortico-tectal loops control the context dependent execution of an eye movement. Therefore, we combined diffusion tensor imaging (DTI) and functional magnetic resonance imaging (fMRI) acquired during the performance of pro- and anti-saccades to determine which of the projections from the FEF to the SC is modulated during antisaccades. The time course of blood-oxygenation-level-dependent (BOLD) activation in FEF-regions projecting to the $\mathrm{CN}$ and SC during pro- and anti-saccades were compared.

To this end the entire FEF as determined using fMRI activation maps was parcellated into different zones based on the white matter tracts uniquely connecting it to the $\mathrm{SC}$ and $\mathrm{CN}$, and event-related BOLD responses in such zones were compared over experimental conditions. Combining fMRI activation patterns and DTI-tractography to investigate the function of connectivity patterns as is demonstrated here might also be a fruitful technique for other fields of neuroscience research.

\section{MATERIALS AND METHODS PARTICIPANTS}

All 15 participants (mean age 23 years, eight males and seven females, all right-handed) were screened for MRI contra-indications. Their vision was normal or corrected to normal. At the beginning of each session the procedures of the experiments were explained, after which a written informed consent was obtained. This study has been approved by the medical ethical commission of the University Medical Center in Utrecht.

\section{APPARATUS}

Imaging was performed on a clinical Philips 3 T Achieva MRI scanner (Philips Medical Systems, Best, Netherlands), equipped with a SENSE coil with eight independent receivers allowing parallel imaging (Pruessmann et al., 1999). Stimuli were projected (using the Presentation software from Neurobehavioral systems) on a Plexiglas $1 \mathrm{~m}$ wide screen placed at $2 \mathrm{~m}$ distance from the participants, and viewed through a mirror mounted on the head coil.

\section{BEHAVIORAL PARADIGMS}

The first task for the participants in the scanner was an eye movement task organized in blocks of eye movements in rapid succession. In this manuscript we refer to this task as the so-called FEF 'localizer'. Block designs yield superior signal detection power as compared to event-related designs (Aguirre and D'Esposito, 2000; Donaldson and Buckner, 2001) and is therefore useful to localize individual FEFs. The localizer task consisted of blocks with pro- and anti-saccades trials in rapid succession. Initially, participants had to fixate a white circle $\left(1^{\circ} \times 1^{\circ}\right.$ visual angle $)$ at the center of a black screen. The circle changes its color to red or blue (the 'cue') and remained visible for $500 \mathrm{~ms}$. The cue color determined 
whether participant needed to perform a pro-saccade (red dot) or an zanti-saccade (blue dot). $300 \mathrm{~ms}$ after disappearance of the cue a peripheral white circle of the same size (as the target) appeared, at $3.8^{\circ}$ or $14.8^{\circ}$ at the left or right side of the center circle. Participants had to make a (pro-)saccade to the target when the cue was red, or to the empty region of the screen exactly opposite of the target when the cue was blue (e.g., the anti-saccade condition). The target remained visible for $950 \mathrm{~ms}$. Subsequently, the white fixation circle reappeared at the center of the screen for $250 \mathrm{~ms}$ to indicate the start of a new trial. A block of 10 such trials lasted 20 s. After each block a center white circle appeared that had to be fixated for $20 \mathrm{~s}$. In total the task lasted $8 \mathrm{~min}$, including 12 saccade and 12 fixation blocks. This task has proven to be successful in robustly activating, among others, the entire zone around the precentral sulcus representing human FEF (Neggers et al., 2007; Van Ettinger-Veenstra et al., 2009). We will define the FEF here as all voxels activated above threshold by this task.

In the next three sessions participants had to perform an eventrelated pro- and anti-saccade task. This task was designed to dissociate BOLD responses from subsequent pro- and anti-saccades. It was identical to the first task, except for larger inter-trial-intervals and there was no explicit rest period. The cue was visible for $3000 \mathrm{~ms}$, the target $6000 \mathrm{~ms}$ and the fixation point, which indicated to participants to make a saccade back to the center of the screen, was visible for $3000 \mathrm{~ms}$ after which a new cycle started. A random interval between +500 and -500 ms was added to the onset of the cue to prevent predictability of the events. For each trial the same random interval was subtracted at the end to assure each trial lasted $12 \mathrm{~s}$. Stimulus dimensions and colors were the same as in the localizer task. In total 40 trials occurred in one session, which lasted $8 \mathrm{~min}$. Three such sessions were conducted consecutively to allow for short rest periods every $8 \mathrm{~min}$, realizing 120 eventrelated trials in total for each participant. Trials were ordered such that five event-related trials were presented together in rows of five pro- or five anti-saccades trials. The direction and eccentricity of the targets was pseudo-randomized from trial to trial, as was the order of the pro- or anti-saccade series of five trials. No eyetracking was performed, as we know from experience outside the MRI that compliance is near perfect for the event-related task as there is a substantial time period between the pro- or anti-saccade cue and the actual saccade target.

\section{IMAGE ACQUISITION}

For each $\mathrm{fMRI}$ session of $8 \mathrm{~min}, 1000$ functional $\mathrm{T}^{*}$ weighted BOLD volumes were acquired using a PRESTO-SENSE acquisition scheme. This method has proven to be more sensitive to signal changes than conventional 2D echo-planar imaging (EPI, Neggers et al., 2008), which is useful for individual FEF localization and event-related response detection. Scan parameters were: $\mathrm{TR}=21.75 \mathrm{~ms}, \mathrm{TE}=32.4 \mathrm{~ms} ; \mathrm{FOV}(\mathrm{ap}, \mathrm{fh}, \mathrm{rl})=224 \mathrm{~mm} \times 256$ $\mathrm{mm} \times 128 \mathrm{~mm}$, flip angle $=10^{\circ}$, matrix: $64 \times 64 \times 32$ slices, voxel size $4 \mathrm{~mm}$ isotropic, 8 -channel head coil, SENSE factor $=2$ and 1.8 (in the left/right and anterior-posterior phase encoding directions, respectively). One volume was acquired in $500 \mathrm{~ms}$ by using parallel imaging in two directions. Note that PRESTO is a pulse sequence that uses 3D acquisition (exciting an entire volume per RF pulse) instead of the $2 \mathrm{D}$ planar acquisition, which is still common in most
fMRI studies that use EPI. Hence TR, which is defined as the time between successive excitations of each proton, equals $21.75 \mathrm{~ms}$. It is sometimes erroneously assumed that TR equals acquisition time per volume, which is only true for 2D EPI (that is still common in $\mathrm{fMRI}$ acquisition). The advantage of $3 \mathrm{D}$ acquisition for event-related fMRI study designs is that, using parallel imaging techniques, it can be accelerated in two phase encoding directions instead of one, allowing the extremely fast acquisition times per volume as used here (Neggers et al., 2008).

For correct coregistration of the functional scans and the T1weighted scan one isolated PRESTO-SENSE volume, the 'reference' or FA25 scan, was acquired with a flip angle of $25^{\circ}$ instead of $10^{\circ}$ leading to more $\mathrm{T} 1$ weighting and hence anatomical contrast. It is otherwise identical to the functional MRI scans.

After functional MRI scanning, a single shot EPI-DTI scan was obtained, consisting of 32 diffusion-weighted scans $\left(b=1000 \mathrm{~s} / \mathrm{mm}^{2}\right)$ with non-colinear gradient directions and one diffusion unweighted scan $\left(b=0 \mathrm{~s} / \mathrm{mm}^{2}\right)$ Scan parameters were: $\mathrm{TR} / \mathrm{TE}=7400 / 70 \mathrm{~ms}$, FOV $240 \mathrm{~mm}$, matrix $128 \times 128,75$ slices thickness $2 \mathrm{~mm}$, no gap, SENSE factor 3, no cardiac gating. Finally, a detailed T1-weighted scan was obtained with parameters $\mathrm{TR} / \mathrm{TE}=9.87 / 4.6 \mathrm{~ms}$, flip angle $=8^{\circ}$, FOV $224 \times 160 \times 168$, matrix $=256 \times 256$, slice thickness $1 \mathrm{~mm}$ (no gap).

\section{DATA ANALYSIS: GENERAL APPROACH}

The main objective of the analyses was to divide the individual FEFs, as identified with the fMRI 'localizer' session, into four different zones: projecting to the ipsilateral CN or SC (for the left and right FEF). This division was based on fiber tracts reconstructed from DTI data that connect the FEF with the SC and CN. Subsequently, event-related BOLD responses following leftward or rightward pro- or anti-saccades are statistically compared for each of these four FEF zones. This was done as follows. First, the functional data from the short FEF 'localizer' task and DTI data (for fiber reconstruction) were analyzed. Second, by combining reconstructed fiber tracts and functional data, FEF voxels could be divided into two groups: uniquely connected to either $\mathrm{CN}$ or SC. For two additional control analyses also FEF zones connected to neither $\mathrm{CN}$ or SC or both were considered. Finally from these different voxel sets an event-related time course was extracted, using data from the three event-related sessions. An estimate of the response amplitudes was used for statistical analysis in order to compare response amplitudes between different FEF zones and conditions. For preprocessing fMRI data SPM5 was used (http://www.fil.ion.ucl. ac.uk/spm/software/spm5/) and matlab routines developed in-house. The details of all the steps are given below.

\section{DATA ANALYSIS: SPATIAL PREPROCESSING}

The T1-weighted anatomical scan and the B0 scan (and all DTI images along with it) were coregistered to the 'reference scan' (a single $\mathrm{T} 2{ }^{*}$ weighted scan with a flip angle of $25^{\circ}$ ) using a rigid body transformation. All four functional sessions were realigned (using the reference scan as the first image) to correct for head movements. Images were resliced at $4 \mathrm{~mm} \times 4 \mathrm{~mm} \times 4 \mathrm{~mm}$ and spatially smoothed with an $8 \times 8 \times 8$ full-width at half maximum Gaussian kernel. All analysis was done in native space, therefore no transformations other then rigid body transformations (such as normalization to MNI stereotactic space) were performed at this stage. 


\section{DATA ANALYSIS: STATISTICAL ANALYSIS OF fMRI DATA}

For the statistical modeling of the 'localizer' task (block design) a general linear model (GLM) with two regressors was fitted to each voxel separately, a common procedure in $\mathrm{fMRI}$ analysis. For the first regressor, a box-car function ( 1 for $20 \mathrm{~s}$ during the task blocks and 0 during 20 s rest blocks) was convolved with the default canonical hemodynamic response function (HRF) from SPM5 (2 weighted gamma functions). The second regressor is a constant modeling baseline. A t-contrast testing the regression coefficient of the task regressor against 0 was calculated (e.g., testing for higher activation during the saccade blocks as compared to fixation), and thresholded at $P<0.05$ corrected for the entire brain volume according to a multiple comparison correction using random field theory (Worsley et al., 1992, 1996).

To isolate individual left and right FEFs, first the activation maps of the 'localizer' task were divided into different sets of connected (e.g., directly neighboring) voxels with supra-threshold $t$-values ('clustering'). This results in a series of volumes with one connected cluster of activated voxels per volume. Next, those maps were selected manually that (at least partially) overlap with the FEF as defined in the volumes of Interest (VOI) Brainmap database (Nielsen and Hansen, 2002). All supra-threshold voxels were substituted with 1 , other voxels with 0 . To accommodate possible inaccuracy, known to occur with fiber tracking because of noise and partial volume error (PVE, Basser et al., 2000; Mori and van Zijl, 2002; Huang et al., 2004), the resulting FEF masks were dilated by one voxel in all directions. The above procedure resulted in a mask in which all clusters not considered to be the FEF were eliminated, but still large enough to catch most fiber tracts coming from subcortical seed areas (see below).

\section{DATA ANALYSIS: DTI FIBER TRACKING}

The DTI\&FiberTools software (Kreher et al., 2006) was used for computation of the tensors and the fractional anisotropy (FA), and for the construction of the white-matter tracts. Fiber tracking was done according the FACT algorithm (Mori et al., 1999), as implemented in the DTI\&FiberTools. Tracking (brute force) was initiated from every voxel in the brain using the standard parameters for the DTI\&fiber toolbox, and at a later stage only fibers passing through the selected CN and SC ROIs were retained (Conturo et al., 1999). Criteria for fiber tracking: as a 'startmask' FA $>0.25$ and mean diffusivity $<0.0016 \mathrm{~mm}^{2} / \mathrm{s}$ were used. As end mask FA $>0.15$ and mean diffusivity $<0.002 \mathrm{~mm}^{2} / \mathrm{s}$ were used. Additional termination criteria were a maximum curvature of the track of $53.1^{\circ}$ and a track traversing less than five voxels. Other studies using the FACT algorithm have used similar tracking parameters (Jiang et al., 2006).

As ROIs, both left and right SC and CN were used. They were manually delineated based on anatomical properties visible on T1-weighted scans. Corresponding locations were projected to the diffusion unweighted (B0) image. We selected $\mathrm{CN}$ and SC anatomically which is feasible due to their clear anatomical contrasts in a T1-weighted scan. It is generally hard to measure fMRI signal change in the SC due to the physiological noise in the midbrain and its large distance to the MR receive coils, especially using highly accelerated $\mathrm{fMRI}$ acquisition as in the current study (Raghunathan, 2004). Furthermore, most likely only parts of the $\mathrm{CN}$ are activated by eye movements (Neggers et al., 2005), at present it is unclear for humans which part for which task. Therefore, we chose to manually delineate the entire $\mathrm{CN}$ and SC on structural scans rather than using functional MR activation patters to not overly bias our results. All fiber bundles were calculated that originate from the SC, left CN and right $\mathrm{CN}$ ROIs in native space images.

Further fiber selection using the aforementioned ROIs and further analyses were done with matlab 2006b scripts developed in-house, see below for details.

\section{DATA ANALYSIS: VOXEL SELECTION}

In the present study event-related BOLD responses to pro- or antisaccades in zones of the FEF uniquely projecting to the SC or the $\mathrm{CN}$ were the main measure of interest. Therefore the left and right FEF volumes as obtained with the fMRI localizer task (see above) were divided into zones projecting to one of these areas as follows.

FEF voxels were selected as belonging to one out of four 'projection classes': uniquely connected to the ipsilateral $\mathrm{CN}$ or SC, to both or to neither $\mathrm{CN}$ nor SC. To be classified as a connected voxel it had to be within an area of $10 \mathrm{~mm}$ from a fiber endpoint originating in the $\mathrm{SC}$ and/or the $\mathrm{CN}$ and positioned in the left or right FEF mask obtained as explained above. We chose this $1-\mathrm{cm}$ distance criterion based on the fact that mainly FA stop criteria cause fibers to terminate at some distance before reaching gray matter, as is well known for FACT fiber tracking (Jones, 2008). The 1-cm criterion should allow fMRI activated voxels in the gray matter to be associated with fibers stopping just before reaching gray matter. With an fMRI-to-fiber selection distance lower than $1 \mathrm{~cm}$ we did not observe one or more projection zones for too many subjects (see also Data Analysis: Data Imputation).

\section{DATA ANALYSIS: EVENT-RELATED TIME COURSE EXTRACTION}

For each voxel in one of the four FEF projection classes, the unprocessed $\mathrm{fMRI}$ time series data was extracted from the three event-related sessions in which participants executed pro- or anti-saccades.

Peri-stimulus time histograms (PSTHs), which can be thought of as averaged event-related responses corrected for overlap of subsequent events which is sometimes referred to as 'selective averaging. PSTHs were created for all voxels within a certain projection class. This is done for leftward and rightward pro- or anti-saccades separately. In order to determine a PSTH, a finite impulse response (FIR) model was fitted to the time series data of each voxel, with the four conditions (pro- or anti-saccade, left- or rightward) modeled separately (window length $12 \mathrm{~s}$ and bin size 0.500 ). The regression coefficients of a FIR model reflect the averaged BOLD signal for a series of time points for a select event type (e.g., leftward, rightward, pro- or anti-saccade), corrected for the overlapping BOLD response (lasting $\sim 12 \mathrm{~s}$ ) of subsequent events, without assuming any predetermined shape of the hemodynamic response (Goutte et al., 2000). To remove low frequency signal drifts, high pass filtering was performed alongside with the FIR modeling using a 128-s cut off frequency. The PSTHs were created with in-house matlab scripts, making use of matlab functions available in the SPM5 matlab code base. Average PSTHs were created from the per-voxel PSTHs for the four saccade types (pro-saccades to the left or right and anti-saccades to the left or right) and four projection zones separately. Note that anti-saccades to the left were defined as 
a saccadic eye movement toward the left, and hence a target located at the right side of the screen. The above procedure was repeated for every participant.

\section{DATA ANALYSIS: STATISTICAL ANALYSIS ON RESPONSE AMPLITUDES}

To perform a statistical analysis on the amplitude of the extracted PSTH profiles the default HRF $h$ from SPM5 (parameters RT $=0.503$, onset delay $4.5 \mathrm{~s}$ and an amplitude of 1 ) is fitted to the individual mean PSTH per condition and projection zone. The function was fitted to the PSTH data using a GLM. The model was constituted of two regressors: the function $h$ discussed above and a constant modeling baseline. The calculated regression coefficient associated with $h$ reflects the estimated amplitude of the event-related fMRI response, comparable to global percentage in signal change.

The individual PSTH amplitudes obtained this way were subjected to an analysis of variance (ANOVA) to statistically test for effects of saccade condition (pro- or anti-saccades, leftward or rightward) and projection class (left or right $\mathrm{CN}$ or SC, both or none).

\section{DATA ANALYSIS: DATA IMPUTATION}

For some participants specific white matter tracts (only for unique connections between the left or right FEF with ipsilateral SC or CN) could not be established using the criteria described above, resulting in missing values in the statistical analysis of the response amplitudes. This was not due to problems with data acquisition (see also the Discussion). Missing tracts were not an issue for projection classes to neither SC nor CN or both. Because each projection is included in four conditions (pro-saccade to the left or right, anti-saccade to the left or right) a missing projection resulted in four missing cells for the ANOVA of that participant. An option was to exclude the participant with one or more missing values which would reduce our sample size to six participants which lacks the statistical power to pick up any difference of interest on the group level. Another possibility is to estimate the missing values based on the data pattern, as missing FEF projection zones were almost equally distributed over subjects (that is, it is not always the same bundle that is missing). The most straightforward way to accomplish this is to use the grand mean of each variable. However, this would artificially lower standard error, possible leading to false positives. Therefore, the missing data pattern was qualified as random missing data (Little and Rubin, 2002), which makes imputation possible and is a common procedure in comparable circumstances. Missing values were imputed by EM algorithm (expectation maximization) with the use of NORM software V2.03 (Schafer, 1999). This algorithm produces maximum likelihood (ML) estimates of the mean values, standard deviation and correlations. Note that we accept only one missing tract out of four to not overly rely on missing value estimation.

For FEF zones uniquely connected to SC or CN, the fitted HRF values were analyzed using SPSS 12 using a $2 \times 2 \times 4$ withinsubjects repeated measures ANOVA. The first factor (condition) has two levels; pro- and anti-saccade. The second factor (direction) has two levels; leftward and rightward. Finally the third factor (projection class) contains four levels, representing the FEF areas connected to ipsilateral SC or $\mathrm{CN}$ : left $\mathrm{CN}$, right $\mathrm{CN}$, left $\mathrm{SC}$ and right SC. Similar ANOVAs were performed for additional analyses of FEF zones connected to both SC and $\mathrm{CN}$, or none (see Results).
On an important note, the comparison of absolute PSTH response amplitudes over FEF zones is not highly informative as they are obtained from clusters in different brain regions consisting of different numbers of voxels. However, differences or interactions with one of the experimental manipulations within a region, and comparisons of these differences with other regions are interpretable as the interactions arise from the same set of voxels.

\section{RESULTS}

\section{LOCALIZATION OF FEF}

The individual FEF could be properly localized using the fast proanti-saccade 'localizer' task. Besides the FEF, parietal and occipital cortices were also activated. The non-FEF clusters were removed from the maps to create FEF masks (see Materials and Methods). One participant showed no evident activation in the FEF and was excluded from further analysis. See Figure 2 for the individual activation maps of four participants. Note the considerable variability in shape, location and size of activated regions over subjects (see also Neggers et al., 2007; Van Ettinger-Veenstra et al., 2009). From these activation maps, the right and left FEF masks were created by assessing the proximity of connected clusters of voxels to known FEF coordinates (Nielsen and Hansen, 2002) (see Materials and Methods).

\section{DTI FIBER TRACKING RESULTS}

For 11 participants at least three out of four tracts could be established. See Figure 3 for an illustrative example of the fibers connecting the CN, SC with the FEF for two such participants. Four participants were excluded from further analyses as less than three of the four intended white matter tracts connecting each FEF with the ipsilateral SC and CN could be isolated.

\section{FEF PARCELLATION INTO PROJECTION ZONES}

After performing the FEF voxel classification according to their projection target (to ipsilateral SC or $\mathrm{CN}$ ), for the 11 remaining participants at least three out of four clusters of voxels were obtained uniquely projecting to either the left and right SC or left and right CN. See Table 1 for an overview of the voxel classification details per participant.

The relative volume of each projection zones was calculated as a percentage of the entire FEF mask (as depicted in Figure 2) on the same side (e.g., for the left and right hemisphere separately). Averaged over subjects, the $\mathrm{CN}-\mathrm{L}$ and $\mathrm{CN}-\mathrm{R}$ projection zones entailed $9.1 \%$ and $10.3 \%$ of the entire FEF volume, the SC$\mathrm{L}$ and SC-R projection zones $10.3 \%$ and $5.0 \%$. Voxels classified as connected to both the ipsilateral $\mathrm{CN}$ and SC occupied $28.0 \%$ and $15.6 \%$ for left and right FEF, and FEF voxels not connected to either CN or SC comprised $52.6 \%$ and $69.1 \%$ for the left and right FEF, respectively.

To investigate whether the FEF projection zones are located on a particular side of the FEF for most participants, the average coordinates of the classified clusters were transformed from native space to MNI stereotactic atlas space (comparable to Talairach). Using the 'unified segmentation' algorithm in SPM5 (Ashburner and Friston, 2005), an inverse normalization warping field was obtained from the high contrast T1-weighted structural scan that can be used to transform individual coordinates to MNI coordinates. See Figure 4 for the layout in MNI space of FEF voxels uniquely projecting to 

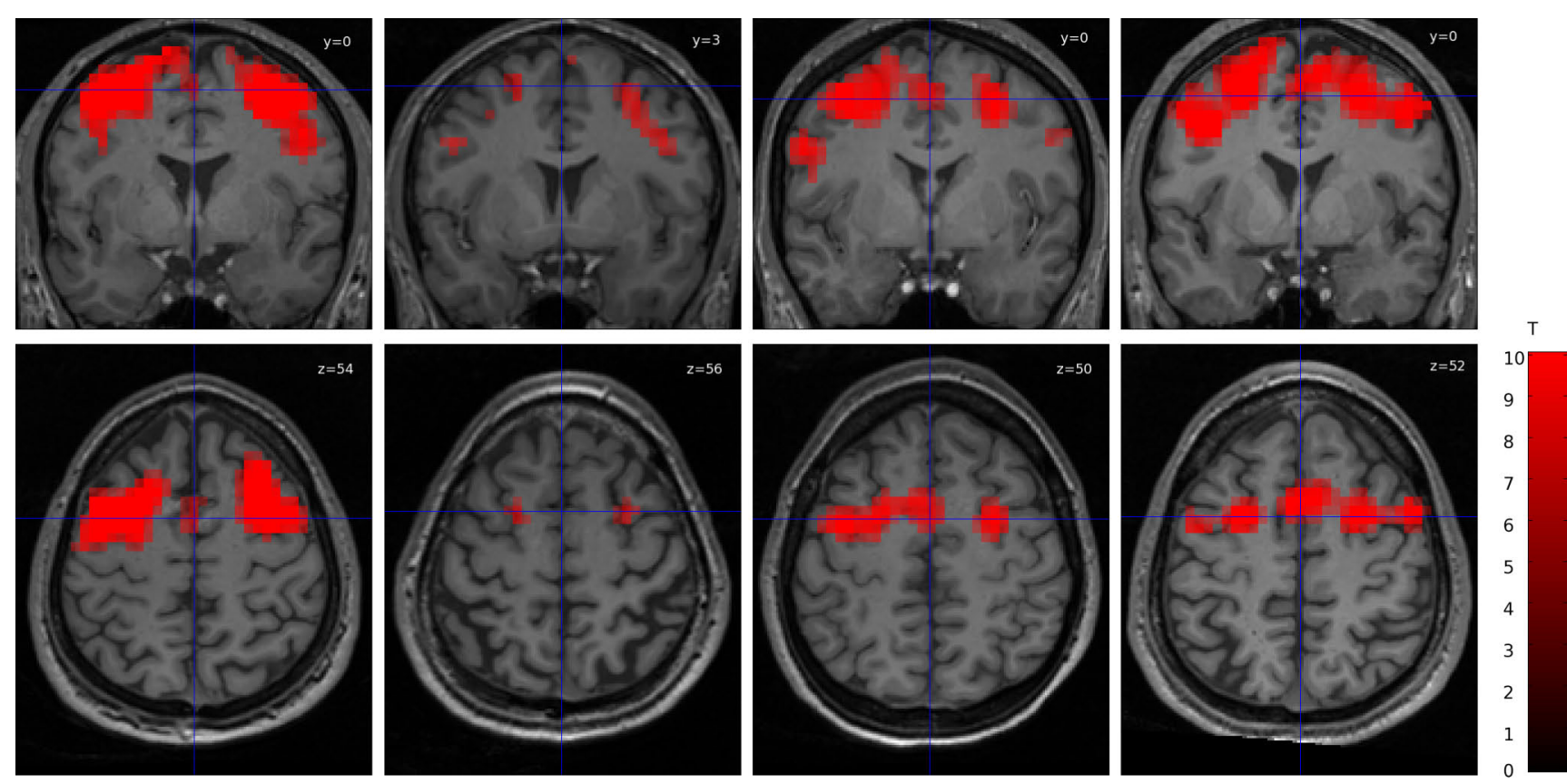

FIGURE 2 | FEF activation t-map for the 'localizer' task in four individual participants at threshold $t>3$, overlaid on theirT1-weighted structural scan, in a coronal (upper panels) and axial (lower panels) view. Only the clusters of activated voxels are shown that overlap with known locations of the

FEF. The data is shown in MNI atlas space and has been normalized from native space only for visualization purposes in this figure. The color codes denote $T$-values for the contrast saccade-block vs rest. For every slice the $y$ and $z \mathrm{MNI}$ coordinate of the cross-section are given in millimeter.

the $\mathrm{CN}$ and the SC from four representative participants, overlaid on a slice (through the center of the cluster of voxels dubbed FEF) from the normalized structural T1-weighted anatomical scan.

The zones in the FEF projecting to the ipsilateral SC were compared with the FEF zones projecting to the $\mathrm{CN}$ in order to establish whether there is a general tendency for specific tracts to originate in a certain part of the FEF. For the left hemisphere, the mean MNI location of the FEF-cluster connected with the $\mathrm{CN}$ was $(-28,1,46)$ and of the cluster connected with the SC $(-22,-2,52)$. In the right hemisphere, the corresponding values were $(25,3,44)$ and $(17,2,58)$, respectively. In the right hemisphere, the zones in the FEF projecting to the $\mathrm{CN}$ tended to be located more laterally; the mean X-coordinate for $\mathrm{CN}$ cluster is significantly larger than the mean $x$-coordinate for the SC-cluster $[T(6)=-2,744, P<0.05]$. Also the FEF zones projecting to the $\mathrm{CN}$ tended to be located more inferior in the right hemisphere, its Z-coordinate is significantly smaller $[T(6)=-2,914$, $P<0.05]$ then for zones projecting to the SC. For the left hemisphere the latter differences were not significant $[T(9)=-1,121, P<0.291$ for $x$-coordinate and $T(9)=-1,009, P<0.339$ for $z$-coordinate]. Note that the $t$-tests were performed on FEF coordinates for only those subjects for which the respective FEF zones could be established.

\section{EVENT-RELATED TIME COURSE}

To investigate the event-related fMRI timecourse for each condition and each FEF projection zone the PSTHs were calculated, see Figure 5. When no white matter tracts were found for a particular participant and projection, no PSTH could be calculated for that FEF subzone for any condition. PSTH calculation for FEF voxels in the left FEF-CN projection class was possible for all 11 participants and for 9 participants for the right FEF-CN projection class. For FEF-SC projection class, this was possible for 10 participants for the left SC and for 9 participants for the right SC, see also Table 1.

Interestingly, FEF responses seem larger in general for antisaccades as compared to pro-saccades. Also, a lateralization of saccade related FEF responses (e.g., the left FEF activating more for saccades to the right and vice versa) as known from single cell recording in non-human primates (Bruce and Goldberg, 1985), was found for the FEF zones projecting to the SC or for FEF zones projecting to both $\mathrm{SC}$ and $\mathrm{CN}$, for anti-saccades only.

Note that the average hemodynamic (e.g., fMRI) response amplitudes (bar height in Figure 5) should not to be compared between FEF zones directly. They are derived from regions with different number of voxels in different parts of the brain; averaging hemodynamic amplitudes over different numbers of voxels for the different zones of the FEF can bias results. The effects of interest are differences of response amplitude between experimental conditions, or interactions thereof, within a region. We assume these differences can then be compared over regions.

For statistical analysis the response amplitude for each PSTH was estimated using a model of the standard HRF from SPM5 (two superimposed gamma functions). These estimated response amplitudes represent the signal change for each condition and projection zone. See Figure 6 for an overview. Response amplitudes for the various conditions were then tested statistically. First a $2 \times 2 \times 4$ within-subjects ANOVA was performed for FEF zones uniquely connected to the $\mathrm{SC}$ or the $\mathrm{CN}$ (data in Figure 6A), missing values had to be estimated for five subjects for whom no DTI connection could be established for at most one out of four FEF zones, see 


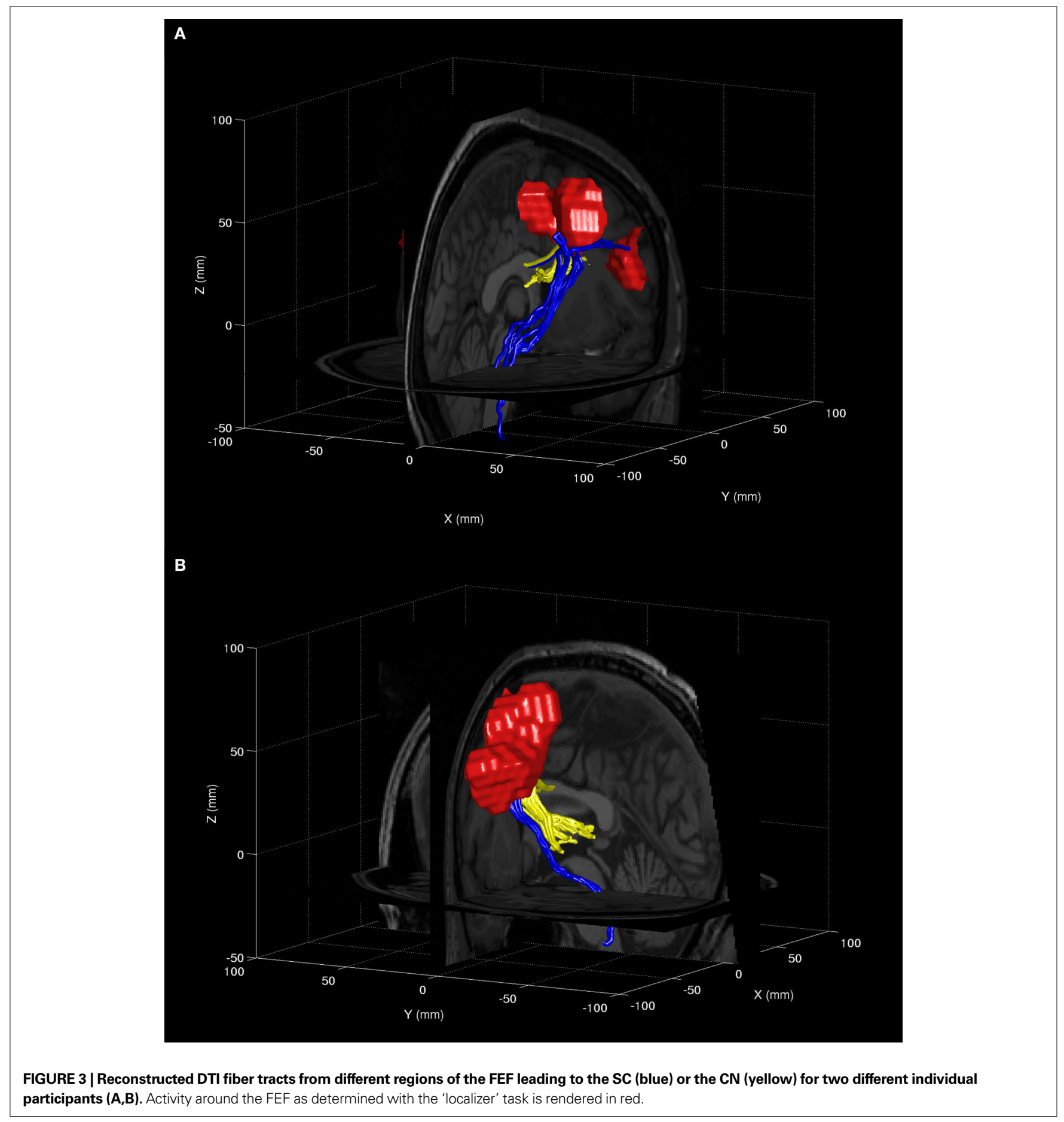

Section 'Data Analysis: Data Imputation' for details. In Table 2 the individual response amplitudes are given for these projection zones and all four conditions. Imputed values are depicted in bold.

A significant main effect for the variable 'condition' was found $[F(1,10)=35.146, P<0.05]$, indicating that the fitted response amplitude is higher in general for anti-saccades as compared to pro-saccades. The within-subjects ANOVA also revealed a three way interaction for 'condition $\times$ projection $\times$ target' $[F(3,30)=3.129$, $P<0.05]$. This implies that the increase in response amplitude for anti-saccades as compared to pro-saccades is mainly observed for FEF zones projecting to the SC, in a lateralized fashion. The latter indicates that the small effects visible in Figure $\mathbf{5}$ are significant.

A post hoc ANOVA on response amplitude in FEF zones projecting to the SC revealed a significant interaction 'target $\times$ projection' $[F(1,10)=8.057, P<0.05]$. In Figure 6A this effect is encircled; it implies that specifically for FEF zones projecting the SC, response amplitudes in the left or right FEF were larger when saccades were made to the contralateral side as compared to the ipsilateral side. 
For the response amplitudes in FEF zones projecting to the $\mathrm{CN}$ this effect was far from significant with $F(1,10)=0.019, P=0.89$. We pooled data for large and small saccade amplitudes as no significant differences were observed.

It should be noted here that averaged over all subjects, the FEF projection zones connected to the $\mathrm{CN}$ were larger than projection zones connected to the SC, both in absolute (number of voxels) and in relative terms (as a percentage of FEF volume), see section 'FEF Parcellation into Projection Zones'. The latter implies that lack of statistical power cannot explain the fact that results were not found for FEF zones connected to the $\mathrm{CN}$, as it comprises averaging over more voxels.

Table 1 |The amount of voxels selected per FEF projection zone, for each participant. The FEF Projection zones are defined as voxels uniquely connected to the ipsilateral caudate nucleus and superior colliculus: $\mathrm{CN} \_$l and SC_I for the left FEF, and CN_r and SC_r for the right FEF. Also, the number of subjects for which those connections could be established is given, as well as the average size of the zones in voxels.

\begin{tabular}{lllll}
\hline Subject From FEF to: & CN_I & CN_r & SC_I & SC_r \\
\hline 1 & 118 & 167 & 0 & 2 \\
2 & 27 & 50 & 1 & 19 \\
3 & 41 & 23 & 6 & 0 \\
4 & 15 & 17 & 82 & 52 \\
5 & 2 & 0 & 76 & 47 \\
6 & 49 & 261 & 44 & 0 \\
7 & 1 & 1 & 140 & 101 \\
8 & 2 & 67 & 13 & 25 \\
9 & 35 & 80 & 44 & 48 \\
10 & 90 & 7 & 4 & 2 \\
11 & 32 & 0 & 10 & 2 \\
No. of subjects & 11 & 9 & 10 & 9 \\
with connections & & & & \\
Average size & 37 & 61 & 38 & 27
\end{tabular}

FIGURE 4 | Spatial distribution of FEF voxels connected to the SC (red circles) or to the $\mathrm{CN}$ (blue circles), in MNI space, plotted in coronal view for four subjects. The circles are projected to a $2 \mathrm{D}$ coronal plane coinciding with a coronal section of the T1 scan normalized to $\mathrm{MNI}$, halfway the selected cluster of

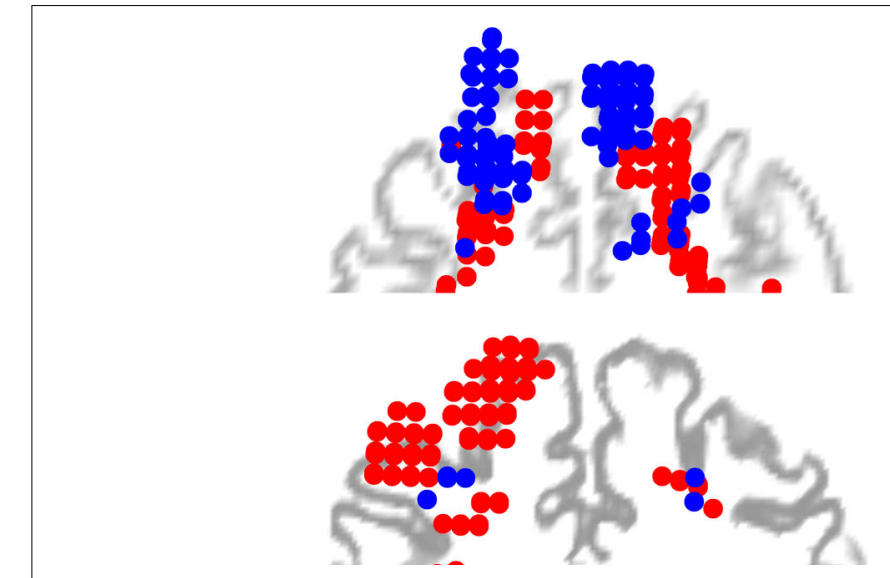

Additionally, we computed PSTHs and response amplitudes for FEF voxels found to be connected to both the $\mathrm{CN}$ and the SC (Figures 5 and 6B). As before, an increase in response amplitude for anti-saccades as compared to pro-saccades was found for FEF zones connected to both the SC and CN [condition: $F(1,10)=31.7$, $P<0.001$ ]. Interestingly, for anti-saccades we found a tendency for the same preference for contralateral saccades as for voxels only connected to the SC, which was significant at trend level in the ANOVA on estimated response amplitudes [projection $\times$ condition: $F(1,10)=3.62, P=0.086]$.

Next, we computed PSTHs for 'unconnected' FEF voxels (e.g., in the localizer maps from Figure 2, but not connected to $\mathrm{CN}$ or SC), see Figure 5. For unconnected voxels, PSTH amplitudes were again larger for anti-saccades in general [condition: $F(1,10)=38.3, P<0.001]$. No other effect was observed for unconnected voxels, e.g., no response was lateralized with respect to saccade direction. This demonstrates that the lateralization with respect to anti-saccade direction found for SC projection zones is specific.

As a final control analysis, we assigned FEF voxels to one of the four projection zones randomly (per FEF, e.g., left FEF voxels were randomly assigned to left $\mathrm{CN}$ or left SC zones, never to right $\mathrm{CN}$ or right SC projection zones). An identical ANOVA as for the $\mathrm{CN}$ and SC projection zones was performed, and again anti-saccades yielded higher response amplitudes [condition: $F(1,10)=36.1, P<0.001]$. No other findings were observed, and response amplitudes were not lateralized with respect to saccade direction.

\section{DISCUSSION}

This study used a combination of fMRI and DTI to disentangle the role of the parallel projections between the FEF and SC in regulating saccades. Both the pathways from FEF to SC as well as to $\mathrm{CN}$ were clearly modulated by task set; for anti-saccades all FEFregions showed a large increase in response amplitude with respect to pro-saccades directed to the same side. From research on non-

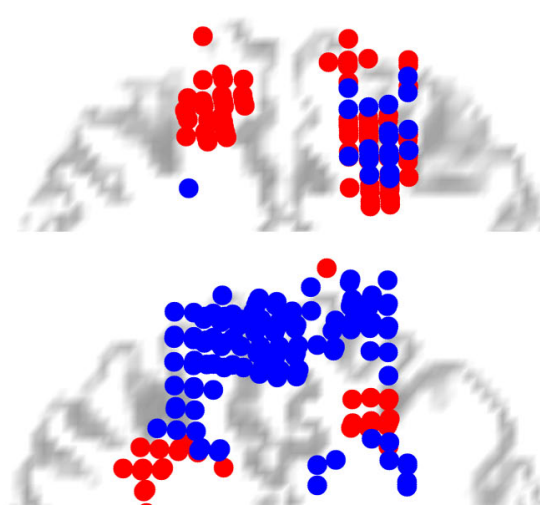

voxels to indicate their position with respect to the individual cerebral anatomy. Note that the distribution of selected FEF voxels entails 3 dimensions, and the T1 coronal section only 2 . This projection is therefore only indicative of the course anatomical location of the voxels and does not exactly match sulcal anatomy. 

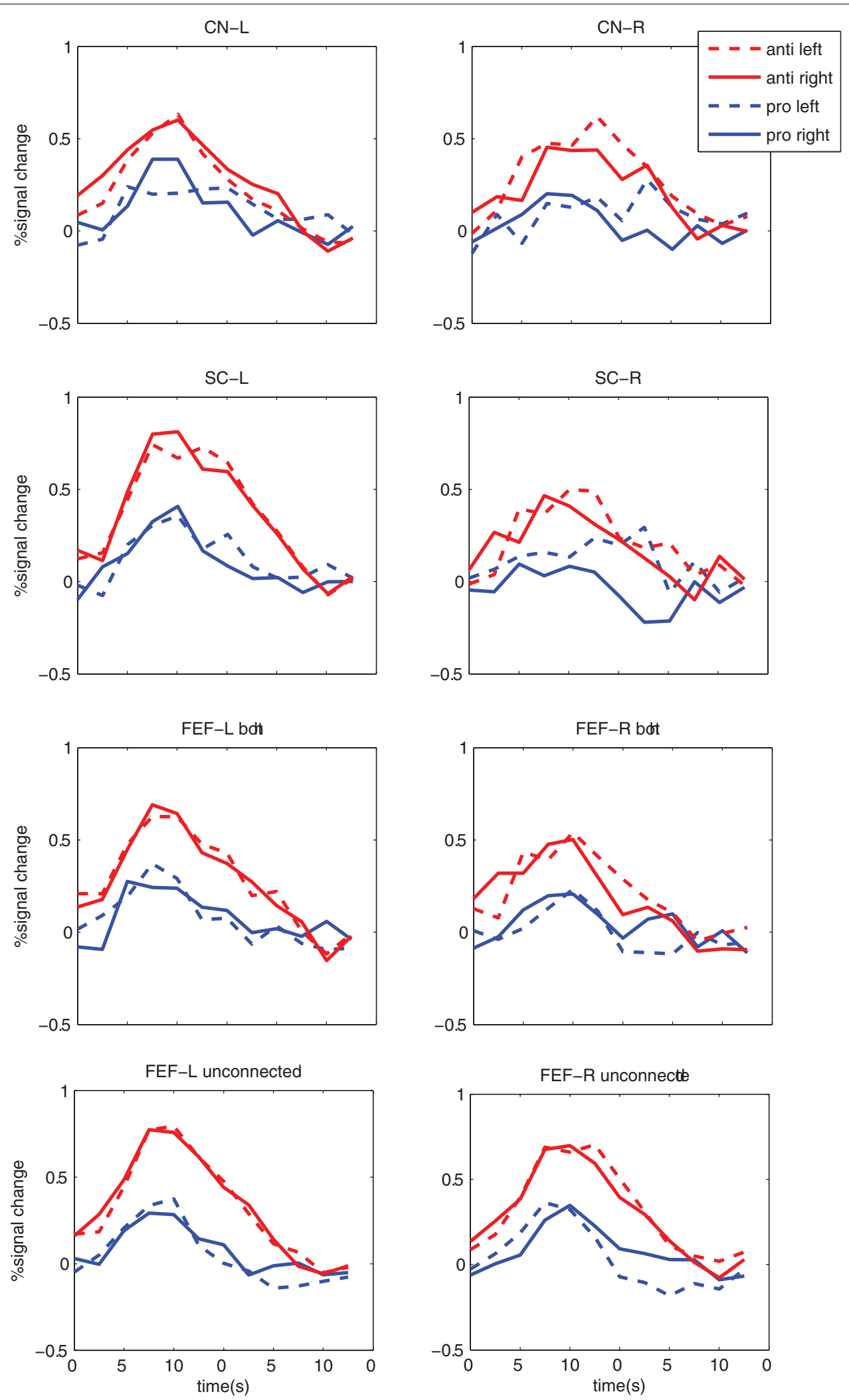

FIGURE 5 | Mean peri-stimulus time histogram (PSTH) of the average BOLD response with respect to time after target presentation for each stimulus condition (pro- or anti, leftward or rightward saccades) and projection

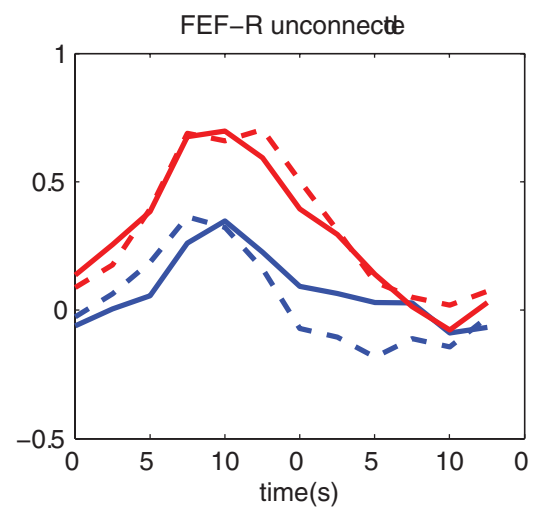

category. CN-L, CN-R (left and right caudate nucleus), SC-L, SC-R (left and right superior colliculus), FEF-L and FEF_R both (FEF voxels connected to both $\mathrm{CN}$ and $\mathrm{SC}$ ), FEF-L and FEF-R unconnected (FEF voxels not connected to $\mathrm{CN}$ nor SC). 

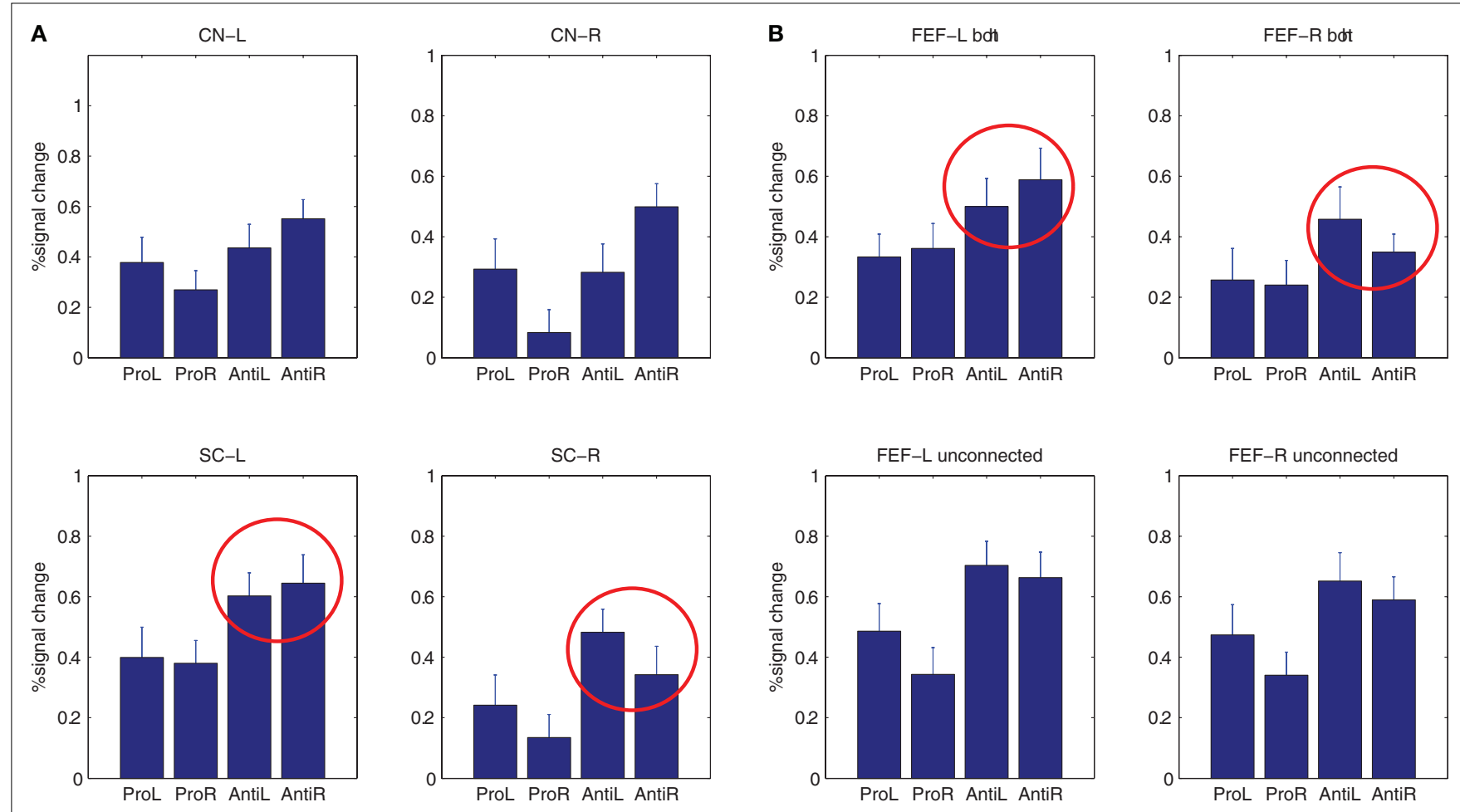

FIGURE 6 | (A) Average hemodynamic response amplitudes of the hemodynamic responses for each condition and FEF zone uniquely connected to the $\mathrm{CN}$ or SC. The lateralization observed for anti-saccades of the response amplitudes with respect to

saccade direction is highlighted with red circles. Error bars denote standard error. (B) Same response amplitudes for FEF zones connected to both the $\mathrm{CN}$ and $\mathrm{SC}$ and for FEF zones connected to neither SC nor CN.

Table 2 | Fitted response amplitudes for FEF zones projecting to either SC or CN and for each condition, for all individual subjects. Due to connections that could not be established (at most 1 per subject, otherwise subject would have been excluded), some of the data had to be estimated (inputted; see Materials and Methods). Estimated values are depicted in bold.

\begin{tabular}{llll}
\hline Area & CN left & CN right & SC left
\end{tabular}

condition

Prol Pro R Antil AntiR ProL ProR Antil AntiR ProL ProR Antil AntiR ProL ProR Pro R AntiR

\begin{tabular}{|c|c|c|c|c|c|c|c|c|c|c|c|c|c|c|c|c|}
\hline Subject 1 & 0.251 & 0.612 & 0.501 & 0.582 & 0.155 & -0.052 & 0.476 & 0.257 & 0.187 & 0.359 & 0.507 & 0.463 & -0.134 & -0.303 & 0.317 & 0.309 \\
\hline Subject 2 & 0.142 & 0.217 & 0.322 & 0.276 & -0.054 & 0.088 & -0.013 & 0.165 & 0.066 & 0.1 & -0.029 & -0.043 & 0.12 & 0.219 & 0.061 & 0.172 \\
\hline Subject 3 & 0.145 & 0.2 & 0.5 & 0.417 & 0.69 & 0.343 & 0.542 & 0.644 & -0.142 & 0.093 & 0.408 & 0.35 & 0.082 & 0.101 & 0.253 & 0.221 \\
\hline Subject 4 & 0.396 & 0.324 & 0.185 & 0.705 & 0.038 & 0.188 & 0.809 & 0.381 & 0.186 & 0.326 & 0.728 & 0.723 & 0.136 & 0.255 & 0.705 & 0.647 \\
\hline Subject 5 & 0.45 & 0.075 & 0.945 & -0.078 & 0.341 & -0.045 & 0.354 & 0.194 & 0.725 & 0.621 & 0.849 & 0.821 & 0.631 & 0.301 & 0.759 & 0.633 \\
\hline Subject 6 & 0.473 & 0.308 & 0.56 & 0.719 & 0.727 & 0.498 & 0.947 & 0.842 & 0.426 & 0.27 & 0.952 & 1.054 & 0.402 & 0.179 & 0.674 & 0.477 \\
\hline Subject 7 & -0.084 & 0.124 & 0.433 & -0.093 & 0.136 & -0.288 & 0.092 & 0.166 & 0.467 & 0.391 & 0.688 & 0.575 & 0.07 & 0.231 & 0.498 & 0.457 \\
\hline Subject 8 & 0.446 & -0.096 & 0.702 & 0.051 & 0.061 & -0.129 & 0.405 & -0.18 & 0.216 & 0.436 & 0.583 & 0.656 & 0.093 & 0.128 & 0.37 & 0.212 \\
\hline Subject 9 & 0.622 & 0.405 & 0.266 & 0.349 & 0.085 & -0.026 & 0.043 & 0.091 & 0.46 & 0.213 & 0.195 & 0.483 & 0.511 & 0.321 & 0.923 & 0.616 \\
\hline Subject 10 & 0.68 & 0.209 & 0.904 & 0.881 & 0.69 & 0.151 & 1.12 & 0.223 & 1.482 & 0.831 & 1.172 & 1.428 & 0.579 & 0.133 & 0.364 & -0.063 \\
\hline Subject 11 & 0.643 & 0.587 & 0.746 & 0.983 & 0.358 & 0.194 & 0.713 & 0.328 & 0.323 & 0.537 & 0.577 & 0.584 & 0.17 & -0.082 & 0.38 & 0.08 \\
\hline
\end{tabular}

human primates, it is known that the FEF represent contralateral saccades (Bruce and Goldberg, 1985). Interestingly, anti-saccade responses in the present study were lateralized in FEF zones connected to the SC or to both the $\mathrm{CN}$ and SC, e.g., larger responses were observed for saccades directed contralaterally. This was not observed for FEF-regions projecting to the $\mathrm{CN}$. The laterality effects were subtle (but significant), which might be due to limited precision of DTI fiber tracking (see also below) and hence averaging of
fMRI responses. Importantly, lack of statistical power as a result of smaller projection zones cannot explain the fact that results were not found for FEF zones connected to the $\mathrm{CN}$, as $\mathrm{CN}$ projection zones were actually larger than SC zones.

In the introduction, we proposed three possible (not mutually exclusive) pathways through which the FEF could enforce anti-saccade execution, as depicted in Figure 1, alongside with predictions for the lateralization of FEF responses with respect to 
saccade direction. For generation of anti-saccades the FEF $>\mathrm{CN}$ pathways, we predicted larger ipsilateral activation of FEF zones connected to the $\mathrm{CN}$ when the indirect pathway is used (Figure 1B) or larger contralateral activation when the direct pathway is used (Figure 1D). None of the latter was observed. When, however, the direct FEF > SC pathway (Figure 1C) is instrumental in antisaccade generation, we predicted larger activation for FEF zones connected to the SC contralateral to saccade direction, which was indeed observed. Based on the present results and assumed model pathways, the pathway from the FEF to the SC from Figure $1 \mathrm{C}$ can therefore be concluded to be more clearly involved in the generation of anti-saccades and/or the inhibition of pro-saccades as compared to the pathways to the $\mathrm{CN}$.

Intriguingly, the same lateralization as in FEF zones connected to the SC was observed (at trend level) for FEF zones connected to both the CN and SC. This could still imply that the connections to the SC mainly contribute to anti-saccade generation, as this lateralization was not observed in FEF zones purely connected to the CN. Due to the limited spatial resolution of both DTI fiber tracking and fMRI, the present results cannot determine whether the effects for FEF zones connected to both CN and SC are due to neurons connected to both regions, to the fact that neurons connected to either the SC or $\mathrm{CN}$ are found in close proximity (e.g., in one fMRI voxel), or to the spatial inaccuracy in DTI fiber tracking leading to overlapping projection zones. The lateralization of FEF responses with respect to saccade direction was not found for voxels connected to neither $\mathrm{CN}$ nor SC, confirming that the structural parcellation of the FEF into projection zones specifically separates FEF function.

We succeeded in localizing two unique regions within both left and right FEF with different functional properties, by determining the white matter tracts connecting the FEF to the SC and CN. This demonstrates that parcellation of a cortical area as defined by fMRI activation patterns using DTI-tractography data is a fruitful technique. Moreover, the parcellation (although rather variable over individuals), exhibited a general pattern: FEF-regions projecting to the $\mathrm{CN}$ tend to be located more inferior and more lateral than the FEF-regions projecting to the SC. This was mainly observed in the right hemisphere.

Another important finding was that for all FEF zones, anti-saccades evoke larger responses than pro-saccades. Several other recent functional imaging studies also reported that in general oculomotor areas in the frontal and parietal cortex exhibit larger responses around anti-saccades as compared to pro-saccades (Connolly et al., 2002; Ford et al., 2005). Clearly, the frontal cortex is involved in the control over automatic in favor of purposeful behavior, as is also known from monkey single cell recordings (Everling and Munoz, 2000), lesion studies (Guitton et al., 1985) and TMS studies (Terao et al., 1998). The FEF zones connected to the SC are not special in this respect, only regarding lateralization with respect to anti-saccade direction they are special.

The role of the many connections between the frontal cortex and the midbrain running through the BG in the control over automatic behavior is still far less clear. It has often been suggested that by means of the inhibitory connections to the SNpr through the $\mathrm{CN}$, the FEF must be in a central position to determine whether the strong tonic inhibition of the SC by the SNpr is released or not (Hikosaka et al., 2000; Munoz and Everling, 2004). Based on the multitude of mostly recurrent pathways through the BG from many cortical areas, some authors also conclude that a key role of the $\mathrm{BG}$ is the selection of an appropriate response from the repertoire of all possible responses (Redgrave et al., 1999), and even that it constitutes a central selection device. This is mostly derived from animal studies, as data on human BG functioning and connectivity is scarce. There is some compelling evidence that indeed the BG are controlling the execution of our responses in a context dependent manner (Vink et al., 2005; Grol et al., 2006). However, none of these studies is combining functional activation data with structural anatomical measures about connectivity such as DTI..

Among the first in vivo reports on human FEF-BG connectivity during anti-saccades, our results suggest that for the oculomotor system, the direct connections between the FEF and SC are involved in anti-saccades to a larger extent than the connections from the FEF to BG. This of course does not suggest that the pathway through the $\mathrm{BG}$ does not play a role in performing anti-saccade tasks as the portions of the FEF connected to the CN did show a general (nonlateralized) increase for anti-saccades.

One can speculate on the function of this increase for the oculomotor pathways through the $\mathrm{CN}$. When performing anti-saccades, a series of processing steps needs to be successfully completed, most likely under control from the FEF. First, the system has to be set for the task by interpreting the instruction. FEF activation differences between pro- and anti-saccades have been reported before saccade target appearance (Helminski and Segraves, 2003), confirming involvement of the FEF in 'preparatory set'. After this, the automatic pro-saccade is to be suppressed and finally, a motor command should be prepared in the opposite direction and then sent to the midbrain, a process referred to as 'vector inversion' (Collins et al., 2008). This command should be stronger than the command needed for the pro-saccade in order to keep the prosaccade suppressed, and hence, lateralized with respect to saccade direction. The latter could be accomplished by the FEF through its direct connections to the SC (Segraves et al., 1987; Stanton et al., 1988), that preserve a contralateral movement field topography (Stanton et al., 1988). This is in agreement with our findings of an increase in BOLD activation for anti-saccades in a lateralized fashion in FEF zones projecting to the SC. Competition within the SC and between the right and left SC, as has been reported for non-human primates (Moschovakis et al., 1988; Munoz and Istvan, 1998), would then resolve the conflict between the desired anti-saccade and the prepotent automatic pro-saccade in favor of the antisaccade. Together with the direct control of anti-saccade execution through direct FEF-SC connections as proposed in Figure 1C, the general suppression of the SC and hence prevention of erroneous pro-saccades mentioned before, presumably slightly earlier in time than actual anti-saccade generation, might be accomplished by FEF pathways involving the inhibitory indirect pathway through the $\mathrm{CN}$ and SNpr. This inhibition of the SC is, however, not necessarily performed ipsilateral to saccade direction as depicted in Figure 1B. Activation of a pathway in one hemisphere during anti-saccades would probably result in bilateral suppression of the SC by the SNpr as has been reported for monkeys (Liu and Basso, 2008). Such a brief suppression of the SC should not occur for pro-saccades, which might explain higher BOLD responses for anti-saccades in general. When one SNpr bilaterally inhibits the SC, as observed for monkeys (Liu and Basso, 2008), one would not necessarily expect lateralization with respect to saccade direction for this pathway, 
which is indeed what we observe for the input signals into the BG pathway from the FEF. One could refer to this putative mechanism as a 'gating' function as it can determine whether a pro-saccade is allowed or not.

The integration of DTI and fMRI data as applied here could be a novel way to delineate functional differences within larger fMRI activation maps, by using structural anatomical information regarding connectivity patterns. However, the present approach clearly has it limitations. First and foremost, current DTI/fiber tracking techniques do not yield precise representations of white matter tracts, but merely a likely location for a set of fibers. Tracking according to the principle direction of diffusion in each voxel is prone to measurement noise in each DTI voxel, is disturbed by crossing fiber tracts (Basser et al., 2000; Mori and van Zijl, 2002; Huang et al., 2004) and depends on tracking algorithm used (Mori and van Zijl, 2002). Specifically, fiber tracking algorithms tend to end shortly before they reach gray matter, as the measured diffusion approaches isotropy there (Jones, 2008). Therefore, we used a $1-\mathrm{cm}$ sphere around each activated voxel to be able classify it to fibers that end before reaching gray matter. Furthermore we incorporated some degree of uncertainty in both the starting regions in the SC and CN as well as in the target region in the FEF as determined using fMRI (e.g., dilated ROIs). The latter approach provides us with only a rough estimate of which parts of the FEF are connected to the $\mathrm{CN}$ and SC, leading to averaging of activation measures over subjects which might explain why our main result, however significant, is small in size. Furthermore, substantial individual variability exists in both the actual FEF location, similar to previous reports (Neggers et al., 2007; Van Ettinger-Veenstra et al., 2009), and in a few participants one of the four fiber bundles of interest could not be isolated. This poses another problem in performing group wise statistics: the inability to reconstruct specific tracts in a minority of participants. This was dealt with by using statistical data imputation (see Materials and Methods and Schafer, 1999; Little and Rubin, 2002). This method estimates the BOLD response at the end of the missing fiber tract (with maximization likelihood estimates) using the response amplitude data for the remaining three tracts together with the values of the other subjects. This is a common and valid method in hypothesis testing using datasets where missing values are inevitable, and can maximize the information one can get out of such incomplete data. Actually, one can bias results when only including participants without any missing values and discarding participants with a missing value, as the underlying reason for missing observations could artificially separate the participants with a missing value from completely observed participants (Raghunathan, 2004). This would complicate extrapolation of the findings to the population from which the sample of subjects was drawn. Furthermore, we only accepted data from subjects where one out of four tracts could not be established, to not overly rely on missing cell estimation.

Note that the fact that sometimes a tract could not be established was not due to problems with data acquisition, the diffusionweighted recordings had normal signal-to-noise ratios for subjects with a tract that could not be established. Rather, this problem is most likely due to the imprecision in the FACT algorithm used for DTI fiber tracking as mentioned before.
One could argue that curvature for DTI fibers leading to more dorsal FEF-regions, as the SC zones we observed, are selected with a bias (e.g., less fibers). Namely, fibers to more dorsal regions might be more curved as to more lateral regions and hence stop earlier due to the maximal curvature tracking parameter used in the FACT fiber tracking algorithm (see Materials and Methods). However, as explained above, we accommodated spatial biases and inaccuracies of fiber tracking by both dilating the FEF ROI as well as $\mathrm{CN}$ and SC masks, and incorporating a $1-\mathrm{cm}$ sphere around an activated fMRI voxel to associate it with a bundle. Finally, the differences in average location between SC and CN zones were rather small. We therefore believe that it is possible that DTI fibers to the more dorsal FEF zone are more often missed due to slightly higher curvature is an unlikely account of our result.

Notwithstanding the challenges mentioned above, we were able to detect subtle but significant differences on a group level between anti- and pro-saccade response characteristics within a single sub-region that was determined using a robust fMRI localizer task and the connectivity patterns with other regions as determined with DTI..

Several recent developments in both fMRI and DTI acquisition and analysis might ameliorate some of the issues we encountered. High-resolution fMRI acquisition techniques $\left(8 \mathrm{~mm}^{3} /\right.$ voxel at $3 \mathrm{~T}$ or $1 \mathrm{~mm}^{3} /$ voxel at $7 \mathrm{~T}$ ) now become available due to parallel imaging (de Zwart et al., 2006) and higher field strengths (Duong et al., 2002; Bodurka et al., 2007). The large activation patterns as determined with conventional fMRI resolution $\left(\sim 64 \mathrm{~mm}^{3} /\right.$ voxel $)$ can, when using high-resolution fMRI, be expected to break up in multiple patches that are more precisely localized which facilitates the classifications of activation patches to certain DTI fiber tracts. Less averaging of the BOLD signal over partially gray and white matter/cerebral spinal fluid voxels has been shown to be beneficial to localization (Duong et al., 2002). Secondly, by using shortened readout epochs, parallel imaging would also decrease the typical spatial EPI distortion (de Zwart et al., 2005) that might have been somewhat different for DTI and fMRI volumes, improving coregistration of both modalities during post processing. Furthermore, the spatial registration of fMRI and DTI data could be improved by scanning DTI twice, with a phase encoding direction running anterior-posterior and vice versa the second time (Andersson et al., 2003). Finally, recent reports suggested fiber tracking algorithms that might be less susceptible to imaging noise and crossing fiber tracts (Mori and van Zijl, 2002), further improving the spatial accuracy.

In summary, the present combined fMRI-DTI study of the human oculomotor system demonstrated that the cortico-tectal pathway from the FEF seems to be most clearly involved in generating anti-saccades. The cortico-striatal pathway from the FEF could have a more general gating function releasing and recovering tonic inhibition of the midbrain by the SNpr at the right moment. The present findings also demonstrate that parcellation of fMRI activation patterns using DTI-fiber tracking can provide new insights in the function of complex neuronal networks in the human brain.

\section{ACKNOWLEDGMENT}

The authors thank Björn Kreher for his support on using DTI\&FiberTools. 


\section{REFERENCES}

Aguirre, G. K., and D'Esposito, M. (2000). "Experimental design for brain fMRI," in Functional MRI, eds C. Moonen and T. W. Bandettini (Heidelberg/Berlin: Springer-Verlag), 369-380.

Alexander, G. E., and Crutcher, M. D. (1990). Functional architecture of basal ganglia circuits: neural substrates of parallel processing. Trends Neurosci. 13, 266-271.

Andersson, J. L., Skare, S., and Ashburner, J. (2003). How to correct susceptibility distortions in spin-echo echo-planar images: application to diffusion tensor imaging. Neuroimage 20, 870-888.

Ashburner, J., and Friston, K. J. (2005). Unified segmentation. Neuroimage 26, 839-851.

Basser, P. J., Pajevic, S., Pierpaoli, C., Duda, J., and Aldroubi, A. (2000). In vivo fiber tractography using DT-MRI data. Magn. Reson. Med. 44, 625-632.

Bodurka, J., Ye, F., Petridou, N., Murphy, K., and Bandettini, P. A. (2007). Mapping the MRI voxel volume in which thermal noise matches physiological noise-implications for fMRI. Neuroimage 34, 542-549.

Bruce, C. J., and Goldberg, M. E. (1985). Primate frontal eye fields. I. Single neurons discharging before saccades. J. Neurophysiol. 53, 603-635.

Collins, T., Vergilino-Perez, D., Delisle, L., and Doré-Mazars, K. (2008).Visual versus motor vector inversions in the antisaccade task: a behavioral investigation with saccadic adaptation. J. Neurophysiol. 99, 2708-2718.

Connolly, J. D., Goodale, M. A., Menon, R.S., and Munoz, D. P. (2002). Human fMRI evidence for the neural correlates of preparatory set. Nat. Neurosci. 5, 1345-1352.

Conturo, T. E., Lori, N. F., Cull, T. S., Akbudak, E., Snyder, A. Z., Shimony, J. S., McKinstry, R. C., Burton, H., and Raichle, M.E. (1999). Tracking neuronal fiber pathways in the living human brain. Proc. Natl. Acad. Sci. U.S.A. 96, 10422-10427.

de Zwart, J. A., Silva, A. C., van, G. P., Kellman, P., Fukunaga, M., Chu, R., Koretsky, A. P., Frank, J. A., and Duyn, J. H. (2005). Temporal dynamics of the BOLD fMRI impulse response. Neuroimage 24, 667-677.

de Zwart, J. A., van Gelderen, P., Golay, X., Ikonomidou, V. N., and Duyn, J. H. (2006). Accelerated parallel imaging for functional imaging of the human brain. NMR. Biomed. 19, 342-351.

Donaldson, D. L., and Buckner, R. L. (2001). "Effective paradigm design," in Functional MRI: An Introduction to Methods, eds P. Jezzard, P. M. Matthews and S. M. Smith (New York: Oxford University Press Inc.), 177-195.
Duong, T. Q., Yacoub, E., Adriany, G., Hu, X., Ugurbil, K., Vaughan, J. T., Merkle, H., and Kim, S. G. (2002) High-resolution, spin-echo BOLD, and CBF fMRI at 4 and 7 T. Magn. Reson. Med. 48, 589-593.

Everling, S., and Munoz, D. P. (2000). Neuronal correlates for preparatory set associated with pro-saccades and anti-saccades in the primate frontal eye field. J. Neurosci. 20, 387-400.

Ford, K. A., Goltz, H. C., Brown, M. R., and Everling, S. (2005). Neural processes associated with antisaccade task performance investigated with event-related FMRI. J. Neurophysiol. 94, 429-440.

Goutte, C., Nielsen, F. A., and Hansen, L. K. (2000). Modeling the haemodynamic response in $\mathrm{fMRI}$ using smooth FIR filters. IEEE Trans. Med. Imaging 19, 1188-1201.

Grol, M. J., de Lange, F. P., Verstraten, F. A., Passingham, R. E., and Toni, I. (2006). Cerebral changes during performance of overlearned arbitrary visuomotor associations. J. Neurosci. 26, 117-125. Guitton, D., Buchtel, H. A., and Douglas, R. M. (1985). Frontal lobe lesions in man cause difficulties in suppressing reflexive glances and in generating goal-directed saccades. Exp. Brain Res. 58, 455-472.

Helminski, J. O., and Segraves, M. A. (2003). Macaque frontal eye field input to saccade-related neurons in the superior colliculus. J. Neurophysiol. 90, 1046-1062.

Hikosaka, O., Takikawa, Y., and Kawagoe, R. (2000). Role of the basal ganglia in the control of purposive saccadic eye movements. Physiol. Rev. 80, 953-978.

Huang, H., Zhang, J., van Zijl, P. C., and Mori, S. (2004).Analysis of noise effects on DTI-based tractography using the brute-force and multi-ROI approach. Magn. Reson. Med. 52, 559-565.

Jiang, H., van Zijl, P. C., Kim, J., Pearlson, G. D., and Mori, S. (2006). DtiStudio: resource program for diffusion tensor computation and fiber bundle tracking. Comput. Methods Programs Biomed. 81, 106-116.

Jones, D. K. (2008). Studying connections in the living human brain with diffusion MRI. Cortex 44, 936-952.

Kreher,B.,Hennig,J., and Il'yasov, K. (2006). DTI\&FiberTools: a complete toolbox for DTI calculation, fiber tracking, and combined evaluation. Proceeding of ISMRM 14th International Scientific Meeting. Seattle, USA.

Little, R. J. A., and Rubin, D. B. (2002). Statistical Analysis with Missing Data, 2nd Edn. New York: Wiley.

Liu, P., and Basso, M.A. (2008). Substantia nigra stimulation influences monkey superior colliculus neuronal activity bilaterally. J. Neurophysiol. 100, 1098-1112.
Mori, S., Crain, B. J., Chacko, V.P., and van Zijl, P. C. (1999). Three-dimensional tracking of axonal projections in the brain by magnetic resonance imaging. Ann. Neurol. 45, 265-269.

Mori, S., and van Zijl, P. C. (2002). Fiber tracking: principles and strategies - a technical review. NMR. Biomed. 15 468-480.

Moschovakis, A. K., Karabelas, A. B., and Highstein, S. M. (1988). Structurefunction relationships in the primate superior colliculus. I. Morphological classification of efferent neurons. $J$. Neurophysiol. 60, 232-262.

Munoz, D. P., and Everling, S. (2004). Look away: the anti-saccade task and the voluntary control of eye movement. Nat. Rev. Neurosci. 5, 218-228.

Munoz, D. P., and Istvan, P.J. (1998). Lateral inhibitoryinteractions in the intermediate layers of the monkey superior colliculus. J. Neurophysiol. 79, 1193-1209.

Neggers, S. F. W., Hermans, E. J., and Ramsey, N. F. (2008). Enhanced sensitivity with fast three-dimensional blood-oxygen-level-dependent functional MRI: comparison of SENSEPRESTO and 2D-EPI at $3 \mathrm{~T}$. NMR Biomed. 21, 663-676.

Neggers, S. F.W., Huijbers, W., Vrijlandt, C. M., Vlaskamp, B. N., Schutter, D. J., and Kenemans, J. L. (2007). TMS pulses on the frontal eye fields break coupling between visuospatial attention and eye movements. J. Neurophysiol. 98, 2765-2778.

Neggers, S. F. W., Raemaekers, M. A. H., Lampmann, E. E. L., Postma, A., and Ramsey, N. F. (2005). Cortical and subcortical contributions to saccade latency in the human brain. Eur. J. Neurosci. 21, 2853-2863.

Nielsen, F. A., and Hansen, L. K. (2002) Modeling of activation data in the BrainMap database: detection of outliers. Hum. Brain Mapp. 15 146-156.

Pruessmann, K.P., Weiger, M., Scheidegger, M. B., and Boesiger, P. (1999). SENSE: sensitivity encoding for fast MRI Magn. Reson. Med. 42, 952-962.

Raghunathan, T. E. (2004). What do we do with missing data? Some options for analysis of incomplete data. Annu. Rev. Public Health 25, 99-117.

Redgrave, P., Prescott, T. J., and Gurney, K (1999). The basal ganglia: a vertebrate solution to the selection problem? Neuroscience 89, 1009-1023.

Schafer, J. L. (1999). NORM: Multiple Imputation of Incomplete Multivariate Data Under a Normal Model [Computer software]. University Park, PA: Department of Statistics, Pennsylvania State University.

Segraves, M. A., Goldberg, M. E., Deng, S. Y., Bruce, C. J., Ungerleider, L. G., and Mishkin, M. (1987). The role of striate cortex in the guidance of eye movements in the monkey. J. Neurosci. 7, 3040-3058.

Sparks, D. L. (2002). The brainstem control of saccadic eye movements. Nat. Rev. Neurosci. 3, 952-964.

Stanton, G. B., Goldberg, M.E., and Bruce, C. J. (1988). Frontal eye field efferents in the macaque monkey: II. Topography of terminal fields in midbrain and pons. J. Comp. Neurol. 271, 493-506.

Terao, Y., Fukuda, H., Ugawa, Y., Hikosaka, O., Hanajima, R., Furubayashi, T., Sakai, K., Miyauchi, S., Sasaki, Y., and Kanazawa, I. (1998). Visualization of the information flow through human oculomotor cortical regions by transcranial magnetic stimulation. J. Neurophysiol. 80, 936-946.

Van Ettinger-Veenstra,H.M.,Huijbers, W., Gutteling, T.P., Vink, M., Kenemans, J. L., and Neggers, S. F. W. (2009). fMRI guided TMS on cortical eye fields: the frontal but not intraparietal eye fields regulate the coupling between visuospatial attention and eye movements. J. Neurophysiol. 102, 3469-3480.

Vink, M., Kahn, R. S., Raemaekers, M., van den, H. M., Boersma, M., and Ramsey, N. F. (2005). Function of striatum beyond inhibition and execution of motor responses. Hum. Brain Mapp. 25, 336-344.

Worsley, K. J., Evans, A. C., Marrett, S., and Neelin, P. (1992). A three-dimensional statistical analysis for $\mathrm{CBF}$ activation studies in human brain. J. Cereb. Blood Flow Metab. 12, 900-918.

Worsley, K. J., Marrett, S., Neelin, P., Vandal, A. C., Friston, K. J., and Evans, A. C. (1996). A unified statistical approach for determining significant signals in images of cerebral activation. Hum. Brain Mapp. 4, 58-73.

Conflict of Interest Statement: The authors declare that the research was conducted in the absence of any commercial or financial relationships that could be construed as a potential conflict of interest.

Received: 10 November 2009; paper pending published: 24 February 2010; accepted: 22 April 2010; published online: 26 May 2010. Citation: de Weijer AD, Mandl RCW, Sommer IEC, Vink $M$, Kahn RS and Neggers SFW (2010) Human fronto-tectal and fronto-striatal-tectal pathways activate differently during antisaccades. Front. Hum. Neurosci. 4:41. doi: 10.3389/fnhum.2010.00041

Copyright (c) 2010 de Weijer, Mandl, Sommer, Vink, Kahn and Neggers. This is an open-access article subject to an exclusive license agreement between the authors and the Frontiers Research Foundation, which permits unrestricted use, distribution, and reproduction in any medium, provided the original authors and source are credited. 